\title{
Dietary inflammatory index in relation to sub-clinical atherosclerosis and atherosclerotic vascular disease mortality in older women
}

\author{
Nicola P. Bondonno ${ }^{1 *}$, Joshua R. Lewis ${ }^{2,3,4}$, Lauren C. Blekkenhorst ${ }^{1}$, Nitin Shivappa ${ }^{5,6,7}$, \\ Richard J. Woodman ${ }^{8}$, Catherine P. Bondonno ${ }^{1,9}$, Natalie C. Ward ${ }^{1,10}$, James R. Hébert ${ }^{5,6,7}$, \\ Peter L. Thompson ${ }^{11}$, Richard L. Prince ${ }^{4,12}$ and Jonathan M. Hodgson ${ }^{1,9}$ \\ ${ }^{1}$ Royal Perth Hospital, School of Medicine and Pharmacology, University of Western Australia, Perth, WA 6000, Australia \\ ${ }^{2}$ Centre for Kidney Research, Children's Hospital at Westmead, Sydney, NSW 2145, Australia \\ ${ }^{3}$ School of Public Health, Sydney Medical School, University of Sydney, Sydney, NSW 2006, Australia \\ ${ }^{4}$ School of Medicine and Pharmacology, Queen Elizabeth Medical Centre, University of Western Australia, Perth, \\ WA 6009, Australia \\ ${ }^{5}$ Cancer Prevention and Control Program, Arnold School of Public Health, University of South Carolina, Columbia, SC 29208, USA \\ ${ }^{6}$ Department of Epidemiology and Biostatistics, Arnold School of Public Health, University of South Carolina, Columbia, SC \\ 29208, USA \\ ${ }^{7}$ Connecting Health Innovations LLC, Columbia, SC 29201, USA \\ ${ }^{8}$ School of Public Health, Centre for Epidemiology and Biostatistics, Flinders University of South Australia, Adelaide, \\ SA 5042, Australia \\ ${ }^{9}$ School of Medical and Health Sciences, Edith Cowan University, Perth, WA 6009, Australia \\ ${ }^{10}$ School of Biomedical Sciences and Curtin Health Innovation Research Institute, Curtin University, Perth, WA 6102, Australia \\ ${ }^{11}$ Department of Cardiovascular Medicine, Sir Charles Gairdner Hospital, Perth, WA 6009, Australia \\ ${ }^{12}$ Department of Endocrinology and Diabetes, Sir Charles Gairdner Hospital, Perth, WA 6009, Australia
}

(Submitted 6 February 2017 - Final revision received 4 May 2017 - Accepted 24 May 2017 - First published online 4 July 2017)

\section{Abstract}

Arterial wall thickening, stimulated by low-grade systemic inflammation, underlies many cardiovascular events. As diet is a significant moderator of systemic inflammation, the dietary inflammatory index (DII ${ }^{\mathrm{TM}}$ ) has recently been devised to assess the overall inflammatory potential of an individual's diet. The primary objective of this study was to assess the association of the DII with common carotid artery-intima-media thickness (CCA-IMT) and carotid plaques. To substantiate the clinical importance of these findings we assessed the relationship of DII score with atherosclerotic vascular disease (ASVD)-related mortality, ischaemic cerebrovascular disease (CVA)-related mortality and ischaemic heart disease (IHD)-related mortality more. The study was conducted in Western Australian women aged over 70 years ( $n$ 1304). Dietary data derived from a validated FFQ (completed at baseline) were used to calculate a DII score for each individual. In multivariable-adjusted models, DII scores were associated with sub-clinical atherosclerosis: a $1 \mathrm{sD}(2 \cdot 13$ units) higher DII score was associated with a 0.013-mm higher mean CCA-IMT $(P=0 \cdot 016)$ and a 0.016-mm higher maximum CCA-IMT $(P=0.008)$, measured at 36 months. No relationship was seen between DII score and carotid plaque severity. There were 269 deaths during follow-up. High DII scores were positively associated with ASVD-related death (per SD, hazard ratio (HR): 1·36; $95 \%$ CI 1.15, 1.60), CVA-related death (per SD, HR: $1.30 ; 95 \%$ CI 1.00, 1.69) and IHD-related death (per SD, HR: 1.40; $95 \%$ CI 1.13, 1.75). These results support the hypothesis that a pro-inflammatory diet increases systemic inflammation leading to development and progression of atherosclerosis and eventual ASVD-related death.

Key words: Dietary inflammatory index: Atherosclerotic vascular disease: Intima-media thickness: Prospective cohort studies

Inflammation is strongly associated with many chronic conditions including $\mathrm{CVD}^{(1)}$. Diet is a significant moderator of systemic inflammation with some foods and nutrients possessing anti-inflammatory properties and others pro-inflammatory properties $^{(2)}$. Diets rich in fruits, vegetables and whole grain foods are linked with lower circulating concentrations of inflammatory biomarkers ${ }^{(3)}$. The dietary inflammatory index $\left(\mathrm{DII}{ }^{\mathrm{TM}}\right.$ ) was created to assess the overall inflammatory potential of an individual's diet by relating particular food intakes to inflammatory biomarkers (C-reactive protein, IL1 $\beta$, IL4, IL6, IL10

Abbreviations: ASVD, atherosclerotic vascular disease; CCA-IMT, common carotid artery-intima-media thickness; CVA, cerebrovascular disease; DII, dietary inflammatory index; HR, hazard ratio; ICD, International Classification of Disease; IHD, ischaemic heart disease.

* Corresponding author: N. P. Bondonno, email nicola.bondonno@uwa.edu.au 
and $\mathrm{TNF}-\alpha)^{(2)}$. It has allowed testing of the hypothesis that more pro-inflammatory diets, indicated by higher DII scores, will be associated with chronic diseases such as $\mathrm{CVD}^{(4)}$. The DII can be calculated from various dietary assessment tools, the most common being $\mathrm{FFQ}^{(2,4,5)}$. Several studies have found positive associations between DII scores and CVD mortality ${ }^{(6-8)}$, however to date no studies have explored the association between the DII and measures of sub-clinical atherosclerosis. Atherosclerosis is the primary cause of $\mathrm{CVD}^{(9)}$ and its development and progression is linked to inflammation ${ }^{(10)}$. Sub-clinical atherosclerosis can be assessed in humans by measuring common carotid artery-intima-media thickness (CCA-IMT) and carotid plaques. Correspondingly, CCA-IMT, and carotid plaques are independently associated with stroke risk ${ }^{(11)}$. CCA-IMT is also an independent predictor of ischaemic heart disease (IHD) ${ }^{(12)}$.

The primary objective of this study was to assess the association of DII scores with CCA-IMT and severe carotid plaques in a cohort of women aged $\geq 70$ years. We also aimed to explore the relationship of DII with atherosclerotic vascular disease (ASVD)-related mortality, and the subgroups ischaemic cerebrovascular disease (CVA)- and IHD-related mortality.

\section{Methods}

\section{Participants}

Data were obtained from a cohort of women aged 70 years and over, who were originally recruited in 1998 to a 5-year, doubleblind, randomised control trial of oral $\mathrm{Ca}$ supplementation to prevent osteoporotic fracture; the Calcium Intake Fracture Outcome Study, which is described in detail elsewhere ${ }^{(13)}$. In brief, 1500 women were recruited from the Western Australian general population by mail using the Electoral Roll, enrolment on which is a requirement of Australian citizenship. All participants were ambulatory, had an expected survival beyond 5 years and were not receiving any medication known to affect bone metabolism. In the subsequent 5 years following inclusion in the study, participants received $1.2 \mathrm{~g}$ of elemental $\mathrm{Ca}$ as calcium carbonate daily or a matching placebo. We have previously shown no effect of Ca treatment on any measure of carotid atherosclerosis in this cohort $^{(14)}$. Participants were followed for a further 10 years as part of the Longitudinal Study of Aging in Women. After excluding participants who did not complete a FFQ at baseline and those with implausible energy intakes $<2100 \mathrm{~kJ} / \mathrm{d}(500 \mathrm{kcal} / \mathrm{d})$ or $>14700 \mathrm{~kJ} / \mathrm{d}$ (3500 kcal/d), 1468 participants remained (Fig. 1). Participants with a history of pre-existing diabetes, myocardial

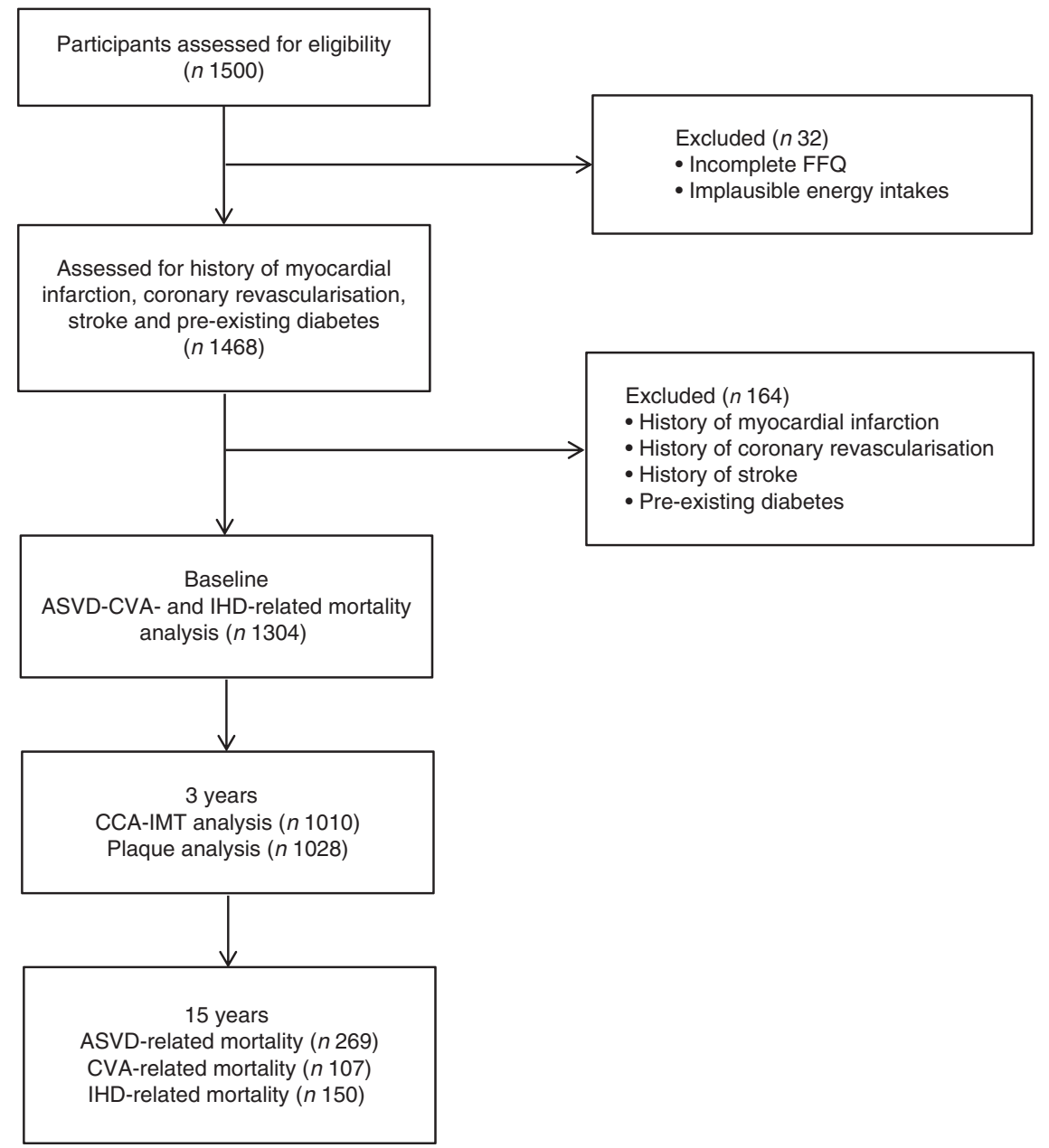

Fig. 1. Consort flow diagram. ASVD, atherosclerotic vascular disease; CVA, cerebrovascular disease; IHD, ischaemic heart disease; CCA-IMT, common carotid artery-intima-media thickness. 
infarction, stroke and/or coronary revascularization were excluded ( $n$ 164), leaving 1304 for the mortality analysis. Of these participants 1010 and 1028 participants had CCA-IMT and plaque measurements taken at 36 months (2001), respectively (Fig. 1).

The participants provided their previous medical history and current medications verified by their General Practitioner. These data were coded using the International Classification of Primary Care-Plus method and were used to determine the presence of pre-existing diabetes (T89001-90009). This coding methodology allows aggregation of different terms for similar pathological entities as defined by the International Classification of Disease (ICD) coding system.

History of myocardial infarction, stroke or coronary revascularisation at baseline was determined using linked data via the Western Australian Data Linkage System. Primary hospital discharge diagnosis codes from 1980-1998 for myocardial infarction included ICD-9-CM code 410 and for stroke included ICD-9-CM codes $430-434^{(15)}$. Coronary revascularisation included coronary artery bypass grafting (CABG); and/or percutaneous transluminal coronary angioplasty (PTCA). CABG procedure codes included: ICD-9-CM codes 36.1-36.19; and Australian classification of health interventions (ACHI) codes 38497, 38500, 38503, 90201 in procedure codes (1-20). PTCA procedure codes included: ICD-9-CM codes 36.0-36.07; and ACHI codes 35310-00, 35310-01, 35310-02, 38306-00, 38306-01, 38306-02, 35304-00, 35305-00, 38300-00, 38303-00, 35335-00, 35338-00, 35338-01, 35341-00, 35344-00, 35344-01, 38309-00, 38312-00, 38312-01, $38315-00$, 38318-00, 38318-01, angioplasty with or without stent on open chest 35310-03, 35310-04, 35310-05, 35304-01, 35305-01, 3850500, 38306-03, 38306-04, 38306-05, 38300-01, 38303-01.

At baseline, written informed consent was obtained from all participants for the study and for the follow-up of electronic health records. The Human Ethics Committee of the University of Western Australia approved the study protocol and consent form (approval no. 05/06/004/H50). The Human Research Ethics Committee of the Western Australian Department of Health also approved the data linkage study (approval no. \#2009/24).

\section{Dietary assessment and calculation of the dietary inflammatory index score}

Baseline (1998) dietary intake was assessed using a validated semi-quantitative FFQ developed by the Cancer Council of Victoria $^{(16)}$. Energy and nutrient intakes were estimated based on frequency of consumption and usual portion size ${ }^{(17)}$. Values from the FFQ were used to calculate a DII score for each individual. The thirty-one food parameters obtained from the FFQ and used to calculate the DII score are presented in Table 1. The DII score is based on a literature review of 1943 articles published up until 2010 linking dietary components to six markers of inflammation. A comprehensive description detailing development of the DII score is available elsewhere ${ }^{(2,18)}$. In brief, to calculate DII score for the participants in this study, the mean intake of every food variable was transformed with standardised values from a
Table 1. Thirty-one food parameters obtained from the FFQ and used to calculate the dietary inflammatory index score

\begin{tabular}{|c|c|}
\hline Food parameters & Overall inflammatory effect score \\
\hline Alcohol (g) & -0.278 \\
\hline$\beta$-Carotene (mg) & -0.584 \\
\hline Carbohydrate (g) & 0.097 \\
\hline Cholesterol (mg) & 0.110 \\
\hline Total fat $(\mathrm{g})$ & 0.298 \\
\hline Fibre $(\mathrm{g})$ & -0.663 \\
\hline Folic acid (mg) & -0.190 \\
\hline Garlic (g) & -0.412 \\
\hline $\mathrm{Fe}(\mathrm{mg})$ & 0.032 \\
\hline $\mathrm{Mg}(\mathrm{mg})$ & -0.484 \\
\hline MUFA (g) & -0.009 \\
\hline Niacin (mg) & -0.246 \\
\hline$n-3$ Fatty acids (g) & -0.436 \\
\hline$n-6$ Fatty acids (g) & -0.159 \\
\hline Onion $(\mathrm{g})$ & -0.301 \\
\hline Protein (g) & 0.049 \\
\hline PUFA (g) & -0.337 \\
\hline Riboflavin (mg) & -0.068 \\
\hline SFA $(g)$ & 0.373 \\
\hline Thiamin (mg) & -0.098 \\
\hline Trans-fat (g) & 0.229 \\
\hline Vitamin A (RE) & -0.401 \\
\hline Vitamin C (mg) & -0.424 \\
\hline Vitamin E (mg) & -0.419 \\
\hline $\mathrm{Zn}(\mathrm{mg})$ & -0.313 \\
\hline Flavan-3-ol (mg) & -0.415 \\
\hline Flavones (mg) & -0.616 \\
\hline Flavonols (mg) & -0.467 \\
\hline Flavonones (mg) & -0.250 \\
\hline Anthocyanidins (mg) & -0.131 \\
\hline Isoflavones (mg) & -0.593 \\
\hline
\end{tabular}

$\mathrm{RE}$, retinol equivalent.

world database to obtain a ' $z$ score'. To minimise the effect of 'right skewing', the ' $z$ ' scores were converted to a proportion and then centred by doubling the proportion and subtracting 1 . This score was then multiplied by the respective food parameter-specific inflammatory effect score derived from the literature review. All of the food parameter-specific DII scores were summed to create the overall DII score for each individual.

\section{Common carotid artery-intima-media thickness and carotid focal plaques}

CCA-IMT and the presence of carotid focal plaques were determined at year 3 (2001) as described in detail elsewhere ${ }^{(19)}$. In brief, six images taken from three different angles (anterolateral, lateral and posterolateral) were examined and the CCAIMT from each image was averaged to give an overall mean CCA-IMT and maximum CCA-IMT. A short-term precision study with repeat IMT measurements yielded a CV of $5.98 \%$, as described previously ${ }^{(20)}$. The complete carotid tree (common carotid artery, carotid bulb, internal and external carotid) was then examined for the presence of focal plaque, defined as an area of focal increased thickness $(\geq 1 \mathrm{~mm})$ of the intima-media layer. Severity of carotid plaque was further dichotomised by the degree of carotid stenosis as either none to minimal $(<25 \%)$ or moderate to high $(\geq 25 \%)^{(21)}$. 
15-year atherosclerotic vascular disease- cerebrovascular disease- and ischaemic heart disease-related mortality

Coded multiple cause of death data over a 15-year period were retrieved from linked mortality data via the Western Australian Data Linkage System. Causes of death were obtained from the coded death certificate using information in Parts 1 and 2 of the death certificate, or all diagnosis text fields from the death certificate where coded deaths were not yet available. ASVD deaths were defined using diagnosis codes from the ICD-9-CM ${ }^{(15)}$ and the International Statistical Classification of Diseases and Related Health Problems, 10th Revision, Australian Modification (ICD-10-AM) ${ }^{(22)}$. ASVD death diagnosis codes included as IHD (ICD-9-CM codes 410-414 and ICD-10-AM codes I20-I25); heart failure (ICD-9-CM code 428 and ICD-10-AM code I50); CVA, excluding haemorrhage (ICD-9-CM codes 433-438 and ICD-10-AM codes I63-I69, G45.9); and peripheral arterial disease (ICD-9-CM codes 440-444 and ICD-10-AM codes I70-I74). Two subgroups of ASVD were also analysed: CVA excluding haemorrhage and IHD (ICD codes as above).

\section{Covariates}

Baseline questionnaires were used to determine values for potential confounding variables including age, socioeconomic status (SES), use of low-dose aspirin, use of antihypertensive medication, use of statins and current or previous smoking. Weight was assessed using digital scales with participants wearing light clothes and no shoes. Height was assessed using a stadiometer and the BMI was calculated in $\mathrm{kg} / \mathrm{m}^{2}$ at baseline. Total energy intakes were estimated from the FFQ. For physical activity, the women filled in a validated questionnaire that allowed estimation of energy used during exercise in $\mathrm{kJ} / \mathrm{d}$ with the use of published energy costs of specific activities ${ }^{(23)}$. The women were asked whether they participated in any sports, recreation or regular physical activity. Women who answered 'no' to this question received a score of 0 and women who answered 'yes' were asked to list up to four forms of regular physical activity undertaken in the past 3 months. Energy expenditure (in $\mathrm{kJ} / \mathrm{d}$ ) for these activities was calculated with the use of published energy costs. SES was estimated from the postal code of the participant's home address. SES was categorised as: 1, top $10 \%$ most disadvantaged; 2, highly disadvantaged; 3, high-medium disadvantaged; 4, medium-low disadvantage; 5, low disadvantage; 6 , top $10 \%$ least disadvantaged. Smoking status was coded as non-smoker or ex-smoker/current smoker (there were only three current smokers) if a participant had ever consumed more than one cigarette per day for more than 3 months during the past. Previous ASVD was determined from primary discharge diagnoses from hospital records (1980-1998) using the ICD codes described previously ${ }^{(24)}$.

\section{Statistical analysis}

A protocol for the statistical analysis of the data was established before the analysis began. Analyses were undertaken using IBM SPSS ${ }^{\circledR}$ Statistics version 21 (2012; IBM Corp.) and SAS ${ }^{\circledR} 9.2$ (SAS Institute). Statistical significance was set at $P \leq 0.05$ (two-tailed) for all tests. DII score was assessed as a continuous variable and a categorical variable when categorised into quartiles: Q1 (-6.140, -1.370); Q2 (-1.371, 0.160); Q3 (0.161, 1.720); Q4 (1.721, 5.800). Descriptive data are presented as mean values and standard deviations for normally distributed continuous variables, medians and interquartile ranges for nonnormally distributed continuous variables and as numbers and percentages for categorical variables. $P$ values for the comparison of baseline characteristics across DII score quartiles were determined by ANOVA or the Kruskal-Wallis $\mathrm{H}$ test for continuous variables or $\chi^{2}$ tests for categorical variables. For all subsequent analyses, two models were fit: (1) age-adjusted only and (2) multivariable-adjusted (age, BMI, energy intake, energy expended in physical activity, SES, use of low-dose aspirin, use of antihypertensive medication, use of statins, current or previous smoking, prevalent ASVD and treatment code). The relationship between mean and maximum CCA-IMT and DII score as a continuous variable was assessed by linear regression using age- and multivariable-adjusted models. We also compared mean and maximum CCA-IMT scores across total DII score quartiles using univariate ANCOVA, with Bonferroni's adjustment for multiple comparisons. The relationship between atherosclerotic plaque severity and DII score, both as a continuous and as a categorical variable, was examined by binary logistic regression using age- and multivariable-adjusted models. Age- and multivariable-adjusted Cox proportional hazard ratios (HR) and 95\% CI of ASVD-, CVA- and IHD-related mortality risk were computed across quartiles of the DII, where the lowest (most anti-inflammatory) quartile was the referent. We tested for evidence of a linear relationship using DII score as a continuous variable in a separate Cox proportional hazards model. Cox proportional hazards assumptions were tested using log-log plots of the survival function $v$. time and assessed for parallel appearance. To assess the extent of possible reverse causality bias, we repeated all analyses after excluding all ASVD, CVA and IHD deaths that occurred within the first 24 months.

\section{Results}

The DII scores were normally distributed, with a mean of $0 \cdot 17$ units and a SD of $2 \cdot 13$ units. The baseline characteristics of the study population overall and stratified by DII score quartiles (Q1 (-6.140, -1.370); Q2 (-1.371, 0.160); Q3 (0.161, 1.720); Q4 $(1 \cdot 721,5 \cdot 800))$ are shown in Table 2 . There were 326 participants in each quartile. At baseline, subjects in the lowest $v$. the highest quartile of DII score had a higher energy intake and a higher level of physical activity. The mean of mean CCA-IMT was $0.779(\mathrm{sD} 0.128) \mathrm{mm}$ (range: $0.423-2.078 \mathrm{~mm}$ ) and the mean of maximum CCA-IMT was 0.923 (SD 0.151$) \mathrm{mm}$ (range: $0.515-2.318 \mathrm{~mm}$ ). A total of $491(47.9 \%)$ participants had a focal plaque present and 137 (13.4\%) participants had moderate to high plaque severity ( $\geq 25 \%$ carotid stenosis).

\section{Dietary inflammatory index and common carotid artery-intima-media thickness}

DII score was linearly associated with mean and maximum CCA-IMT in age and multivariable-adjusted models (Table 3). 
Table 2. Baseline characteristics of study population, stratified by dietary inflammatory index (DII) quartiles (Q), Longitudinal Study of Aging in Women, Western Australia, 1998-2013

(Mean values and standard deviations; medians and interquartile ranges (IQR); mean values and standard deviations)

\begin{tabular}{|c|c|c|c|c|c|c|c|c|c|c|c|}
\hline \multirow[b]{3}{*}{ Characteristics } & & & \multicolumn{9}{|c|}{ DII quartiles } \\
\hline & \multicolumn{2}{|c|}{ Total } & \multicolumn{2}{|c|}{ Q1* } & \multicolumn{2}{|c|}{ Q2* } & \multicolumn{2}{|c|}{$\mathrm{Q3}^{*}$} & \multicolumn{2}{|c|}{ Q4* } & \multirow[b]{2}{*}{$P+$} \\
\hline & $n$ & $\%$ & $n$ & $\%$ & $n$ & $\%$ & $n$ & $\%$ & $n$ & $\%$ & \\
\hline Age (years) & & & & & & & & & & & 0.54 \\
\hline Mean & \multicolumn{2}{|c|}{$75 \cdot 1$} & \multicolumn{2}{|c|}{$75 \cdot 3$} & \multicolumn{2}{|c|}{$75 \cdot 0$} & \multicolumn{2}{|c|}{$75 \cdot 0$} & \multicolumn{2}{|c|}{$75 \cdot 2$} & \\
\hline $\mathrm{SD}$ & \multicolumn{2}{|c|}{$2 \cdot 7$} & \multicolumn{2}{|c|}{2.6} & \multicolumn{2}{|c|}{$2 \cdot 7$} & \multicolumn{2}{|c|}{$2 \cdot 7$} & \multicolumn{2}{|c|}{$2 \cdot 8$} & \\
\hline BMI $\left(\mathrm{kg} / \mathrm{m}^{2}\right)$ & & & & & & & & & & & 0.78 \\
\hline Mean & \multicolumn{2}{|c|}{$27 \cdot 0$} & \multicolumn{2}{|c|}{$27 \cdot 1$} & \multicolumn{2}{|c|}{$26 \cdot 8$} & \multicolumn{2}{|c|}{$27 \cdot 1$} & \multicolumn{2}{|c|}{$27 \cdot 0$} & \\
\hline $\mathrm{SD}$ & \multicolumn{2}{|c|}{$4 \cdot 6$} & & & \multicolumn{2}{|c|}{$4 \cdot 3$} & \multicolumn{2}{|c|}{4.6} & \multicolumn{2}{|c|}{$5 \cdot 0$} & \\
\hline Energy intake $(1000 \mathrm{~kJ} / \mathrm{d})$ & & & & & & & & & & & 0.01 \\
\hline Mean & \multicolumn{2}{|c|}{$7 \cdot 1$} & \multicolumn{2}{|c|}{$9 \cdot 0$} & & & & & & & \\
\hline $\mathrm{SD}$ & & & & & & & & & & & \\
\hline Physical activity $(100 \mathrm{~kJ} / \mathrm{d})$ & & & & & & & & & & & 0.01 \\
\hline Median & & & & & & & & & & & \\
\hline IQR & & & & & & & & & & & \\
\hline Socioeconomic status & & & & & & & & & & & 0.99 \\
\hline Bottom $10 \%$ & 46 & $3 \cdot 6$ & 13 & $4 \cdot 1$ & 12 & $3 \cdot 7$ & 10 & $3 \cdot 1$ & 11 & 3.4 & \\
\hline Low & 154 & 11.9 & 36 & $11 \cdot 3$ & 35 & $10 \cdot 8$ & 36 & $11 \cdot 1$ & 47 & 14.5 & \\
\hline Low-medium & 210 & $16 \cdot 2$ & 53 & $16 \cdot 6$ & 56 & $17 \cdot 2$ & 53 & $16 \cdot 4$ & 48 & $14 \cdot 8$ & \\
\hline Medium-high & 198 & $15 \cdot 3$ & 50 & $15 \cdot 6$ & 50 & $15 \cdot 4$ & 51 & $15 \cdot 8$ & 47 & 14.5 & \\
\hline High & 268 & $20 \cdot 7$ & 67 & $20 \cdot 9$ & 68 & $20 \cdot 9$ & 62 & $19 \cdot 2$ & 71 & $21 \cdot 8$ & \\
\hline Top $10 \%$ & 417 & $32 \cdot 3$ & 101 & 31.6 & 104 & $32 \cdot 0$ & 111 & 34.4 & 101 & $31 \cdot 1$ & \\
\hline Low-dose aspirin use & 232 & $17 \cdot 8$ & 64 & $19 \cdot 6$ & 43 & $13 \cdot 2$ & 61 & $18 \cdot 7$ & 64 & $19 \cdot 6$ & 0.09 \\
\hline Antihypertensive use & 537 & $41 \cdot 2$ & 140 & 42.9 & 122 & $37 \cdot 4$ & 139 & $42 \cdot 6$ & 136 & $41 \cdot 7$ & 0.45 \\
\hline Statin use & 209 & $16 \cdot 0$ & 63 & $19 \cdot 3$ & 46 & $14 \cdot 1$ & 47 & $14 \cdot 4$ & 53 & $16 \cdot 3$ & 0.24 \\
\hline Smoked ever & 470 & $36 \cdot 3$ & 121 & $37 \cdot 3$ & 116 & $35 \cdot 7$ & 116 & 35.9 & 117 & $36 \cdot 1$ & 0.97 \\
\hline Prevalent ASVD & 88 & 6.7 & 21 & $6 \cdot 4$ & 20 & $6 \cdot 1$ & 26 & $8 \cdot 0$ & 21 & 6.4 & 0.78 \\
\hline Ca treatment & 677 & $52 \cdot 0$ & 179 & 54.9 & 175 & $53 \cdot 8$ & 164 & $50 \cdot 3$ & 159 & $48 \cdot 8$ & 0.35 \\
\hline
\end{tabular}

ASVD, atherosclerotic vascular disease. Quartiles of DII: Q1 (-6.140, -1.370); Q2 (-1.371, 0.160); Q3 (0.161, 1.720); Q4 (1.721, 5.800).

${ }^{*} n 326$.

$\dagger P$ value for the comparison between DII quartiles, by ANOVA, Kruskal-Wallis $\mathrm{H}$ test or $\chi^{2}$ test where appropriate.

Table 3. Associations between dietary inflammatory index, measured at baseline, and mean and maximum common carotid arteryintima-media thickness (CCA-IMT) measured at year 3, Longitudinal Study of Aging in Women, Western Australia, 1998-2013* (Unstandardised coefficients and $95 \%$ confidence intervals)

\begin{tabular}{|c|c|c|c|c|c|c|}
\hline \multirow[b]{2}{*}{ Characteristics } & \multicolumn{3}{|c|}{ Age adjusted } & \multicolumn{3}{|c|}{ Multivariable adjusted $\dagger$} \\
\hline & $B$ & $95 \% \mathrm{Cl}$ & $P$ & $B$ & $95 \% \mathrm{Cl}$ & $P$ \\
\hline \multicolumn{7}{|l|}{ CCA-IMT } \\
\hline Mean (per 1 SD) & 0.012 & $0.004,0.020$ & 0.002 & 0.013 & $0.002,0.023$ & 0.016 \\
\hline Maximum (per 1 SD) & 0.015 & $0.006,0.024$ & 0.002 & 0.016 & $0.004,0.028$ & 0.008 \\
\hline
\end{tabular}

A 1 SD $(2 \cdot 13$ units $)$ higher DII score was associated with a 0.013-mm (95\% CI 0.002, 0.023) higher mean CCA-IMT and a 0.016-mm (95\% CI 0.004, 0.028) higher maximum CCA-IMT after multivariable adjustment for pre-specified baseline risk factors.

The effect of diet-mediated inflammation was further explored by assessing mean and maximum CCA-IMT across DII score quartiles (Fig. 2). Participants with a DII score $>1.721$ (top quartile) had a significantly higher CCA-IMT than participants with a DII score $\leq 1.370$ (bottom quartile) after multivariable adjustment for pre-specified baseline risk factors ((mean differences; mean CCA-IMT: $0.039 \mathrm{~mm}$; 95\% CI 0.002, 0.077, $P=0.03$; maximum CCA-IMT: $0.047 \mathrm{~mm}$; $95 \%$ CI $0.003,0.091$, $P=0.03)$ ).

\section{Dietary inflammatory index score and carotid focal plaques}

Data were available on carotid focal plaques for 1026 participants, of which $13.6 \%$ had moderate to severe carotid stenosis ( $\geq 25 \%$ ). Neither DII score as a continuous nor as a categorical covariate was a predictor of plaque severity in age- and multivariable-adjusted models (Table 4).

\section{Dietary inflammatory index score and 15-year} atherosclerotic vascular disease- cerebrovascular disease- and ischaemic heart disease-related mortality

During 16947 person-years of follow-up, 269 out of 1304 (20.7\%) participants died from an ASVD-related cause. Of these, 107 were related to ischaemic CVA and 150 were related 
(a)

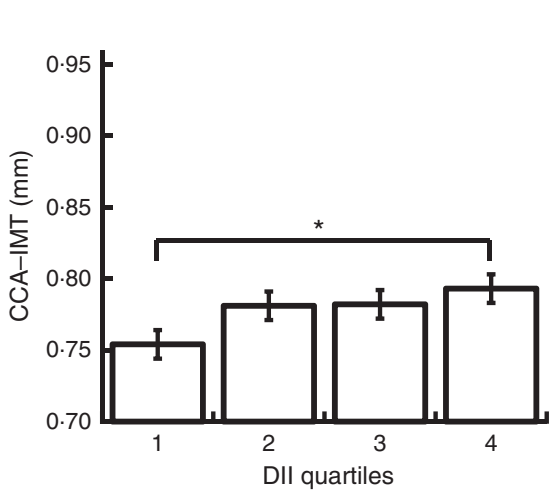

(b)

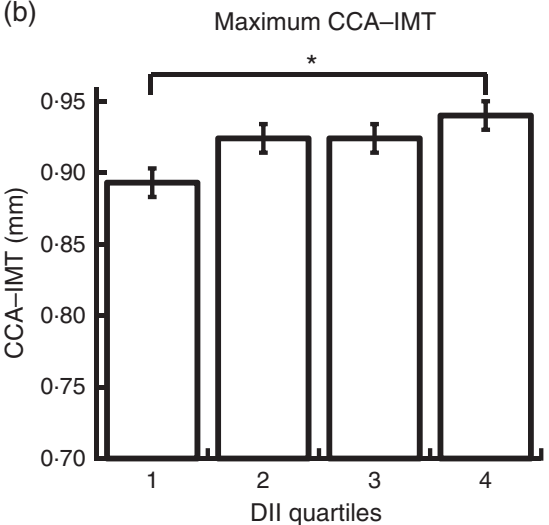

Fig. 2. Mean (a) and maximum (b) common carotid artery-intima-media thickness (CCA-IMT) by quartiles (Q) of dietary inflammatory index (DII): Q1 (-6.140, -1.370); Q2 (-1.371, 0.160); Q3 (0.161, 1.720); Q4 (1.721, 5.800). Values are means, with their standard errors represented by vertical bars analysed by ANCOVA and adjusted for age, BMI, energy intake, energy expended in physical activity, socioeconomic status, use of low-dose aspirin, use of antihypertensive medication, use of statins, current or previous smoking, prevalent atherosclerotic vascular disease and treatment. Linear trend was assessed by a linear regression model (a: $P=0.01$; b: $P<0.01)$. ${ }^{*}$ Significantly different with Bonferroni adjustment for multiple comparisons $(P<0.05)$.

Table 4. Associations between dietary inflammatory index (DII), measured at baseline, and moderate-severe atherosclerotic plaque measured at year 3 , Longitudinal Study of Aging in Women, Western Australia, 1998-2013

(Odds ratios and $95 \%$ confidence intervals)

\begin{tabular}{|c|c|c|c|c|c|c|c|c|}
\hline \multirow[b]{2}{*}{ Characteristics } & \multirow[b]{2}{*}{ Cases $(n)$} & \multirow[b]{2}{*}{ Non-cases $(n)$} & \multicolumn{3}{|c|}{ Age adjusted } & \multicolumn{3}{|c|}{ Multivariable adjusted* } \\
\hline & & & OR & $95 \% \mathrm{Cl}$ & $P$ & OR & $95 \% \mathrm{Cl}$ & $P$ \\
\hline Continuous DII score & 137 & 889 & 1.00 & $0.92,1.09$ & 0.94 & 1.00 & $0.90,1.12$ & 0.95 \\
\hline DII Q1 & 36 & 228 & 1.00 & Ref. & & 1.00 & Ref. & \\
\hline DII Q2 & 32 & 227 & 0.90 & $0.54,1.50$ & 0.69 & 0.98 & $0.56,1.7$ & 0.94 \\
\hline DII Q3 & 34 & 222 & 0.99 & $0.60,1.63$ & 0.96 & 0.99 & $0.54,1.81$ & 0.97 \\
\hline DII Q4 & 35 & 212 & 1.04 & $0.63,1.72$ & 0.88 & 1.09 & $0.56,2.09$ & 0.81 \\
\hline
\end{tabular}

Ref., referent values. Quartiles (Q) of DII: Q1 (-6.140, -1.370); Q2 (-1.371, 0.160); Q3 (0.161, 1.720); Q4 (1.721, 5.800).

* Binary logistic regression models were multivariable adjusted for age, BMI, energy intake, energy expended in physical activity, socioeconomic status, use of low-dose aspirin, use of antihypertensive medication, use of statins, current or previous smoking, prevalent atherosclerotic vascular disease and treatment.

to IHD. Age-adjusted and multivariable-adjusted associations between DII score and mortality are shown in Table 5. For every $1 \mathrm{sD}(2 \cdot 13$ units) increase in DII score, there was an associated $36 \%$ higher risk of ASVD-related death (HR: 1.36; $95 \%$ CI $1.15,1.60)$ in the multivariable-adjusted model. Similarly, in multivariable-adjusted models, for every $1 \mathrm{SD}$ increase in DII score, there was an associated 30 and $40 \%$ higher risk of CVA-related mortality (HR: $1.30 ; 95 \%$ CI 1.00, 1.69) and IHDrelated mortality (HR: 1.40; $95 \%$ CI $1.13,1.75$ ), respectively.

After adjustments for confounding risk factors, participants in the highest (most pro-inflammatory) quartile of DII score were at a higher risk of an ASVD- (HR: 2.02; $95 \%$ CI 1.30, 3.13), CVA(HR: $1.76 ; 95 \%$ CI $0.92,3.40$ ) or IHD-related (HR: $2.51 ; 95 \% \mathrm{CI}$ $1 \cdot 37,4.62$ ) cause of death in comparison to those in the lowest quartile of the DII score, although this did not reach significance for CVA-related mortality $(P=0 \cdot 09)$. The multivariable-adjusted survival outcomes for ASVD, CVA and IHD according to quartiles of DII score are presented in Fig. 3(a)-(c).

\section{Sensitivity analyses}

Dietary inflammatory index score and 15-year atherosclerotic vascular disease-related mortality. To assess the extent of reverse causality bias, we excluded all ASVD, CVA and IHD deaths that occurred within the first 24 months. This did not significantly attenuate the relationship between DII score and ASVD mortality (HR: $1.34 ; 95 \%$ CI $1 \cdot 14,1 \cdot 58, P=0.001$ ), CVA mortality (HR: $1.30 ; 95 \%$ CI 1.00, 1.69, $P=0.049)$ and IHD mortality (HR: 1.37; $95 \%$ CI: $1 \cdot 10,1 \cdot 71, P=0 \cdot 005)$.

\section{Discussion}

In the present cohort of postmenopausal women we have shown for the first time that a pro-inflammatory diet may be associated with an increase in a marker of carotid atherosclerosis (CCA-IMT), as well as increased relative hazards of 15-year ASVD, and IHDrelated mortality in women over the age of 70 years with high DII score. There was also a borderline significant association $(P=0.05)$ between DII and CVA-related mortality.

As postmenopausal women are at a higher risk of CVD than premenopausal women ${ }^{(25)}$, it is critical to investigate potential modifiable risk factors for CVD in such a population. Diet is one of the most important and potentially modifiable of these risk factors. A Western-style diet, recognised to be rich in red and processed meats, sweets, French fries and refined grains, is positively associated with inflammatory biomarkers ${ }^{(26)}$, whereas a Mediterranean-style diet, characterised by a high consumption of olive oil, whole grains, fruits, vegetables, nuts and 
Table 5. Associations between dietary inflammatory index (DII) and mortality from multivariate Cox proportional hazards models, Longitudinal Study of Aging in Women, Western Australia, 1998-2013

(Hazards ratios (HR) and $95 \%$ confidence intervals)

\begin{tabular}{|c|c|c|c|c|c|c|c|c|}
\hline \multirow[b]{2}{*}{ Characteristics } & \multirow[b]{2}{*}{$n(\%)$} & \multirow[b]{2}{*}{ Person-years } & \multicolumn{3}{|c|}{ Age adjusted } & \multicolumn{3}{|c|}{ Multivariable adjusted ${ }^{*}$} \\
\hline & & & $\mathrm{HR}$ & $95 \% \mathrm{Cl}$ & $P$ & $\mathrm{HR}$ & $95 \% \mathrm{Cl}$ & $P$ \\
\hline \multicolumn{9}{|l|}{ ASVD mortality } \\
\hline Continuous DII score $†$ & $20 \cdot 7$ & 16947 & 1.24 & $1.10,1.41$ & $<0.01$ & 1.36 & $1.15,1.60$ & $<0.01$ \\
\hline DII Q1 & $16 \cdot 9$ & 4368 & 1.00 & Ref. & $<0.01 \ddagger$ & 1.00 & Ref. & $<0.01 \ddagger$ \\
\hline DII Q2 & $18 \cdot 7$ & 4193 & 1.22 & $0.85,1.75$ & & 1.31 & $0.90,1.93$ & \\
\hline DII Q3 & 21.5 & 4314 & 1.36 & $0.95,1.93$ & & $1 \cdot 39$ & $0.92,2.12$ & \\
\hline DII Q4 & $25 \cdot 5$ & 4072 & 1.72 & $1.22,2.42$ & & $2 \cdot 02$ & $1.30,3.13$ & \\
\hline \multicolumn{9}{|l|}{ CVA mortality } \\
\hline Continuous DII score & 8.2 & 16947 & 1.20 & $0.99,1.46$ & 0.07 & 1.30 & $1.00,1.69$ & 0.05 \\
\hline DII Q1 & 8.6 & 4368 & 1.00 & Ref. & $0.08 \ddagger$ & 1.00 & Ref. & $0.07 \ddagger$ \\
\hline DII Q2 & $5 \cdot 2$ & 4193 & 0.66 & $0.36,1.21$ & & 0.73 & $0.39,1.37$ & \\
\hline DII Q3 & $8 \cdot 0$ & 4314 & 0.99 & $0.58,1.68$ & & 1.08 & $0.57,2.04$ & \\
\hline DII Q4 & $1 \cdot 1$ & 4072 & 1.46 & $0.89,2.40$ & & 1.76 & $0.92,3.40$ & \\
\hline \multicolumn{9}{|l|}{ IHD mortality } \\
\hline Continuous DII score & 11.5 & 16947 & $1 \cdot 16$ & $0.99,1.37$ & 0.07 & 1.40 & $1.13,1.75$ & $<0.01$ \\
\hline DII Q1 & 8.9 & 4368 & 1.00 & Ref. & $0.07 \ddagger$ & 1.00 & Ref. & $<0.01 \ddagger$ \\
\hline DII Q2 & $12 \cdot 2$ & 4193 & 1.52 & $0.94,2.45$ & & 1.87 & $1 \cdot 13,3 \cdot 10$ & \\
\hline DII Q3 & $12 \cdot 6$ & 4314 & 1.52 & $0.94,2.44$ & & 1.96 & $1.11,3.45$ & \\
\hline DII Q4 & $12 \cdot 3$ & 4072 & 1.58 & $0.98,2.56$ & & $2 \cdot 51$ & $1.37,4.62$ & \\
\hline
\end{tabular}

ASVD, atherosclerotic vascular disease; Ref., referent values; CVA, cerebrovascular disease; IHD, ischaemic heart disease. Quartiles of DII: Q1 (-6.140, -1.370$)$; Q2 (-1.371, 0.160); Q3 (0.161, 1.720); Q4 (1.721, 5.800)

* Cox proportional hazards models were multivariable adjusted for age, BMI, energy intake, energy expended in physical activity, socioeconomic status, use of low-dose aspirin, use of antihypertensive medication, use of statins, current or previous smoking, prevalent ASVD and treatment.

† Calculated per SD (2.13 units) increase in DII.

$\ddagger P$ value for trend.

fish, is linked with lower levels of inflammatory biomarkers ${ }^{(3)}$. Investigating the inflammatory effects of individual foods or isolated nutrients can misrepresent in vivo effects, as these constituents are usually ingested along with other foods or as part of a whole food matrix. In this study, the DII score was used to reflect the overall inflammatory potential of the participants' diet. Previous studies have demonstrated significant positive associations between DII score and other inflammatory biomarkers such as high-sensitivity $\mathrm{CRP}^{(5,18,27)}$ and IL-6 ${ }^{(5,18,28,29)}$.

Arterial wall thickening, stimulated by low-grade systemic inflammation, underlies many CVD events ${ }^{(9)}$. CCA-IMT, a measure of arterial wall thickness, is an independent predictor of $\mathrm{CHD}^{(12)}$ and stroke ${ }^{(30)}$, even after adjustment for traditional risk factors. To our knowledge, this is the first study to explore the relationship of DII score with sub-clinical atherosclerosis. We demonstrated that a $1 \mathrm{SD}$ increase in DII score was associated with a $0 \cdot 013-\mathrm{mm}$ higher mean CCA-IMT and a 0.016-mm higher maximum CCA-IMT. The observed differences in CCA-IMT may be clinically important as a $0.03-\mathrm{mm}$ increase per year in CCA-IMT confers a relative risk of $2 \cdot 2$ (95\% CI 1.4, 3.6) for a coronary event ${ }^{(31)}$.

In the present study, the risk of cardiovascular mortality increased progressively with each quartile of DII score. In contrast to our multivariable-adjusted model results showing an increase in the risk of ASVD- CVA- and IHD-related mortality, a similar analysis performed in a population of middle-aged Australian women produced null results ${ }^{(32)}$. In that study there was a suggestion of increased risk with a higher DII score and the authors suggest that the large CI indicated a lack of power rather than a lack of association. In the Seguimiento Universidad de Navarra (SUN) cohort in Spain, the multivariable-adjusted HR for cardiovascular events (myocardial infarction, stroke or cardiovascular death) for participants in the highest $v$. the lowest quartile of the DII score was $2 \cdot 03$ (95\% CI 1.06, 3.88) ${ }^{(33)}$. This association was attenuated and became non-significant after excluding participants with chronic aspirin intake. In the present study, multivariable adjustment included aspirin intake and did not significantly weaken the observed relationships between DII score and mortality. Three other prospective cohort studies by Shivappa et al. have found positive associations between DII score and CVD mortality ${ }^{(6-8)}$.

Strengths of the present study include its prospective design with extended period of follow-up and use of the literaturederived DII which has been validated with a variety of inflammatory markers ${ }^{(5,18,28,29)}$. We had access to detailed information on lifestyle and cardiovascular risk factors for study participants as well as a validated measure of our primary outcome, CCA-IMT. Furthermore, adjusting for a large range of confounding factors did not significantly attenuate the observed relationship between DII and CCA-IMT.

We also acknowledge several limitations of our study. The number of deaths experienced was relatively low. Although we were able to detect a relationship between DII score and ASVDand IHD-related mortality, the limited number of CVA-related deaths may have impaired our ability to detect significant differences between the highest and lowest quartile of DII score. Second, the DII score was calculated from only one FFQ, which does not provide us with information on any change in dietary pattern throughout follow-up. We recognise that the evidence for causality for the relationship of DII with CCA-IMT in this study is weakened by not having CCA-IMT measures at the same time as the DII assessment. In this regard, the association 
between the two measures (DII and CCA-IMT) more closely resembles evidence from a cross-sectional design rather than a study prospective design. A strong correlation was found between the DII score calculated from the baseline FFQ and the
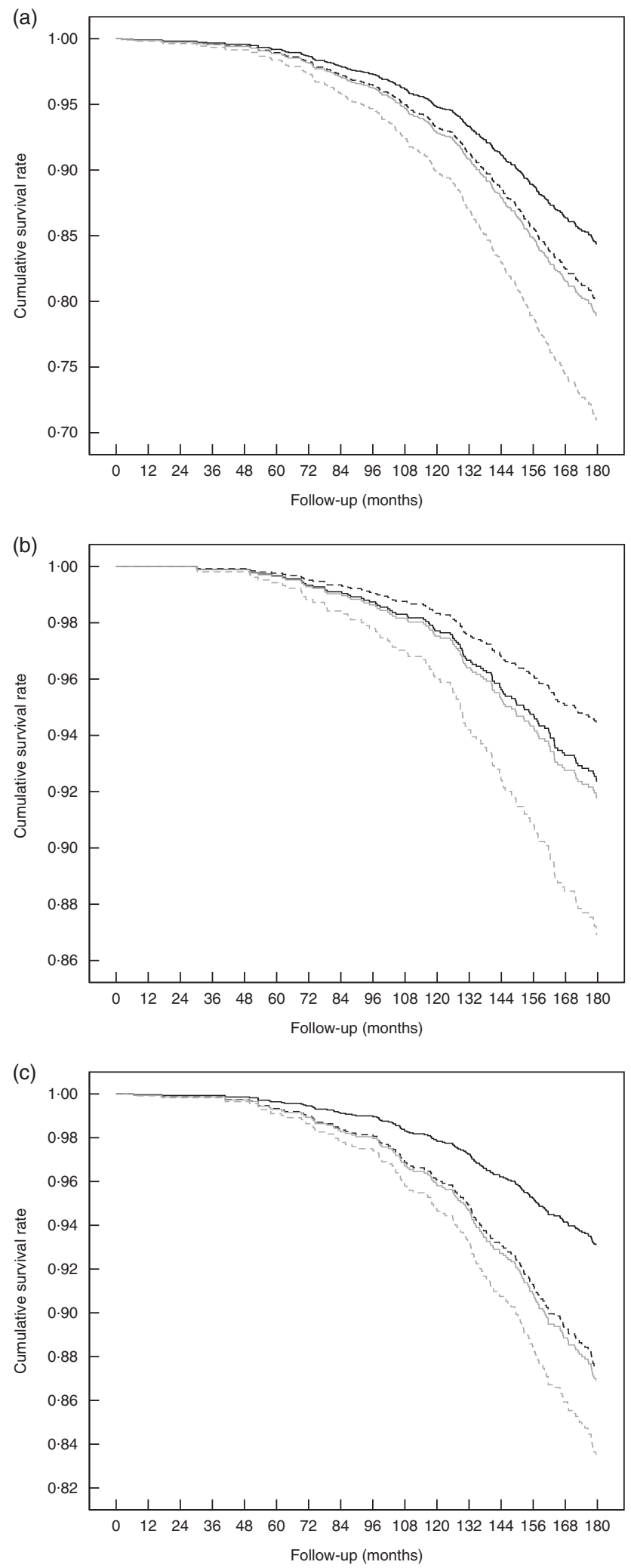

DII score calculated from a second FFQ completed at 60 months $(r 0.51, P<0 \cdot 001)$. Given that CCA-IMT is a measure of generalised atherosclerosis, a strong predictor of ASVD mortality, and DII was associated with both CCA-IMT and ASVD deaths; it is most likely that direction of the association between DII and the atherosclerotic process was causal rather than either inverse or bi-directional. Finally, the present study population was limited to women over the age of 70 years and caution should be taken when extrapolating these results to men and younger women.

\section{Conclusions}

In the present cohort we found that postmenopausal women who consumed a more pro-inflammatory diet, reflected by a higher DII score were at an increased risk of dying from ASVD, specifically ischaemic CVA or IHD. We also have shown for the first time that a pro-inflammatory diet is linearly associated with sub-clinical atherosclerosis. This supports the hypothesis that a pro-inflammatory diet increases systemic inflammation leading to the development and progression of atherosclerosis. This could then result in a higher risk of mortality from heart disease or stroke.

\section{Acknowledgements}

The authors wish to thank the staff at the Data Linkage Branch, Hospital Morbidity Data Collection and Registry of Births, Deaths and Marriages for their work on providing data for this study.

The study was supported by Kidney Health Australia grant S07 10, Healthway Health Promotion Foundation of Western Australia, Sir Charles Gairdner Hospital Research Advisory Committee Grant and by project grants 254627, 303169 and 572604 from the National Health and Medical Research Council of Australia. Hologic Inc. provided the software for image review. The salary of J. R. L. is supported by a National Health and Medical Research Council of Australia Career Development Fellowship (ID: 1107474). The salary of J. M. H. is supported by a National Health and Medical Research Council of Australia Senior Research Fellowship. N. S. and J. R. H. were supported by the US National Institute for Diabetes, Digestive and Kidney Diseases (grant no. R44DK103377). None of the funding agencies had any role in the conduct of

Fig. 3. (a) Atherosclerotic vascular disease ( $n$ 269), (b) ischaemic cerebrovascular disease ( $n$ 107) and (c) ischaemic heart disease ( $n$ 150) survival outcomes for quartiles (Q) of dietary inflammatory index (DII). Multivariable-adjusted Cox regression model included age, BMI, energy intake, energy expended in physical activity, socioeconomic status, use of low-dose aspirin, use of antihypertensive medication, use of statins, current or previous smoking, prevalent atherosclerotic vascular disease and treatment. HR, hazard ratios; a: — DII Q1 (-6.140, -1.370) - referent; - - - -, DII Q2 (-1.371, 0.160) - HR 1.31; 95\% Cl 0.90, 1.93, $P=0.16$; - . - -, DII Q4 (1.711, 5.800) - HR 2.02; 95\% Cl 1.30, 3.13, P<0.01. b: —, DII Q1 (-6.140, -1.370) - referent; - - - -, DII Q2 (-1.371, 0.160) - HR 1.73; 95\% Cl $0.39,1.37, P=0.32 ;-$, DII Q3 $(0.161,1.710)-\mathrm{HR} 1.08 ; 95 \% \mathrm{Cl} 0.57,2.04$, $P=0.81$; - - - , DII Q4 (1.711, 5.800) - HR 1.76; 95\% Cl 0.92, 3.04, $P=0.09$. c: — DII Q1 (-6.140, -1.370) - referent; - - - -, DII Q2 (-1.371, 0.160) - HR 1.87; 95\% Cl 1.13, 3.10, P=0.02; — DII Q3 $(0.161,1.710)-\mathrm{HR} 1.96 ; 95 \% \mathrm{Cl}$ 1.11, 3.45, $P=0.02$; - - - , DII Q4 (1.711, 5.800) - HR 2.51; 95\% Cl 1.37, 4.62, $P<0.01$. 
the study; collection, management, analysis or interpretation of the data; or preparation, review or approval of the manuscript.

N. P. B., J. R. L, N. S., L. C. B., R. L. P. and J. M. H. were responsible for the project conception; J. R. L. and R. L. P. collected the data; N. P. B., J. R. L., L. C. B., R. J. W., R. L. P. and J. M. H. developed the research plan; N. S. and J. R. H. contributed expertise and algorithms to calculate the DII scores and consulted on DII-related analyses. N. P. B., R. J. W. and J. M. H. analysed the data; N. P. B. and J. M. H. prepared the manuscript; J. R. L., N. S., L. C. B., R. J. W., C. P. B., J. R. H., P. L. T. and R. L. P. critically reviewed the manuscript.

J. R. H. owns controlling interest in Connecting Health Innovations (CHI) LLC, a company planning to license the right to his invention of the DII from the University of South Carolina in order to develop computer and smart phone applications for patient counselling and dietary intervention in clinical settings. N. S. is an employee of CHI.

The authors declare that there are no conflicts of interest.

\section{References}

1. Pearson TA, Mensah GA, Alexander RW, et al. (2003) Markers of inflammation and cardiovascular disease application to clinical and public health practice: a statement for healthcare professionals from the centers for disease control and prevention and the American Heart Association. Circulation 107, 499-511.

2. Shivappa N, Steck SE, Hurley TG, et al. (2014) Designing and developing a literature-derived, population-based dietary inflammatory index. Public Health Nutr 17, 1689-1696.

3. Esposito K, Marfella R, Ciotola M, et al. (2004) Effect of a Mediterranean-style diet on endothelial dysfunction and markers of vascular inflammation in the metabolic syndrome: a randomized trial. JAMA 292, 1440-1446.

4. Tabung FK, Steck SE, Zhang J, et al. (2015) Construct validation of the dietary inflammatory index among postmenopausal women. Ann Epidemiol 25, 398-405.

5. Shivappa N, Steck SE, Hurley TG, et al. (2014) A populationbased dietary inflammatory index predicts levels of C-reactive protein in the Seasonal Variation of Blood Cholesterol Study (SEASONS). Public Health Nutr 17, 1825-1833.

6. Shivappa N, Blair CK, Prizment AE, et al. (2016) Association between inflammatory potential of diet and mortality in the Iowa Women's Health Study. Eur J Nutr 55, 1491-1502.

7. Shivappa N, Harris H, Wolk A, et al. (2016) Association between inflammatory potential of diet and mortality among women in the Swedish Mammography Cohort. Eur J Nutr 55, 1891-1900

8. Shivappa N, Steck SE, Hussey JR, et al. (2017) Inflammatory potential of diet and all-cause, cardiovascular, and cancer mortality in National Health and Nutrition Examination Survey III Study. Eur J Nutr 56, 683-692.

9. Burke GL, Evans GW, Riley WA, et al. (1995) Arterial wall thickness is associated with prevalent cardiovascular disease in middle-aged adults the atherosclerosis risk in communities (ARIC) study. Stroke 26, 386-391.

10. Hansson GK (2005) Inflammation, atherosclerosis, and coronary artery disease. N Eng J Med 352, 1685-1695.

11. Touboul P-J, Labreuche J, Vicaut E, et al. (2005) Carotid intima-media thickness, plaques, and Framingham risk score as independent determinants of stroke risk. Stroke 36, 1741-1745.
12. Chambless LE, Heiss G, Folsom AR, et al. (1997) Association of coronary heart disease incidence with carotid arterial wall thickness and major risk factors: the Atherosclerosis Risk in Communities (ARIC) Study, 1987-1993. Am J Epidemiol 146, 483-494.

13. Prince RL, Devine A, Dhaliwal SS, et al. (2006) Effects of calcium supplementation on clinical fracture and bone structure: results of a 5-year, double-blind, placebo-controlled trial in elderly women. Arch Intern Med 166, 869-875.

14. Lewis JR, Zhu K, Thompson PL, et al. (2014) The effects of 3 years of calcium supplementation on common carotid artery intimal medial thickness and carotid atherosclerosis in older women: an ancillary study of the CAIFOS randomized controlled trial. J Bone Miner Res 29, 534-541.

15. World Health Organization (1977) Manual of the International Statistical Classification of Diseases, Injuries and Causes of Death, 9th Revision (ICD-9). Geneva: WHO.

16. Hodge A, Patterson AJ, Brown WJ, et al. (2000) The Anti Cancer Council of Victoria FFQ: relative validity of nutrient intakes compared with weighed food records in young to middle-aged women in a study of iron supplementation. Aust N Z J Public Health 24, 576.

17. Ireland P, Jolley D, Giles G, et al. (1994) Development of the Melbourne FFQ: a food frequency questionnaire for use in an Australian prospective study involving an ethnically diverse cohort. Asia Pac J Clin Nutr 3, 19-31.

18. Cavicchia PP, Steck SE, Hurley TG, et al. (2009) A new dietary inflammatory index predicts interval changes in serum highsensitivity C-reactive protein. J Nutr 139, 2365-2372.

19. Ivey KL, Lewis JR, Hodgson JM, et al. (2011) Association between yogurt, milk, and cheese consumption and common carotid artery intima-media thickness and cardiovascular disease risk factors in elderly women. Am J Clin Nutr 94, 234-239.

20. Bonnick SL, Johnston CC, Kleerekoper M, et al. (2001) Importance of precision in bone density measurements. J Clin Densitom 4, 105-110.

21. Wilson PW, Hoeg JM, D'Agostino RB, et al. (1997) Cumulative effects of high cholesterol levels, high blood pressure, and cigarette smoking on carotid stenosis. The New England journal of medicine 337, 516-522.

22. National Centre for Classification in Health (1998) International Statistical Classification of Diseases and Related Health Problems, 10th Revision, Australian Modification (ICD-10-AM). Sydney: National Centre for Classification in Health.

23. Bruce DG, Devine A \& Prince RL (2002) Recreational physical activity levels in healthy older women: the importance of fear of falling. J Am Geriatr Soc 50, 84-89.

24. Lewis JR, Lim W, Dhaliwal SS, et al. (2012) Estimated glomerular filtration rate as an independent predictor of atherosclerotic vascular disease in older women. BMC Nephrol 13, 58.

25. Gorodeski GI (2002) Update on cardiovascular disease in post-menopausal women. Best Pract Res Clin Obstet Gynaecol 16, 329-355

26. Lopez-Garcia E, Schulze MB, Fung TT, et al. (2004) Major dietary patterns are related to plasma concentrations of markers of inflammation and endothelial dysfunction. Am J Clin Nutr 80, 1029-1035.

27. Wirth MD, Burch J, Shivappa N, et al. (2014) Association of a dietary inflammatory index with inflammatory indices and metabolic syndrome among police officers. J Occup Environ Med 56, 986-989.

28. Wood LG, Shivappa N, Berthon BS, et al. (2015) Dietary inflammatory index is related to asthma risk, lung function and systemic inflammation in asthma. Clin Exp Allergy 45 , 177-183. 
29. Shivappa N, Hébert JR, Rietzschel ER, et al. (2015) Associations between dietary inflammatory index and inflammatory markers in the Asklepios Study. Br J Nutr 113, 665-671.

30. Chambless LE, Folsom AR, Clegg LX, et al. (2000) Carotid wall thickness is predictive of incident clinical stroke: the Atherosclerosis Risk in Communities (ARIC) study. Am J Epidemiol 151, 478-487.

31. Hodis HN, Mack WJ, LaBree L, et al. (1998) The role of carotid arterial intima-media thickness in predicting clinical coronary events. Ann Intern Med 128, 262-269.
32. Vissers LET, Waller MA, van der Schouw YT, et al. (2016) The relationship between the dietary inflammatory index and risk of total cardiovascular disease, ischemic heart disease and cerebrovascular disease: findings from an Australian population-based prospective cohort study of women. Atherosclerosis 253, 164-170.

33. Ramallal $\mathrm{R}$, Toledo E, Martínez-González MA, et al. (2015) Dietary inflammatory index and incidence of cardiovascular disease in the SUN cohort. PLOS ONE 10, e0135221. 